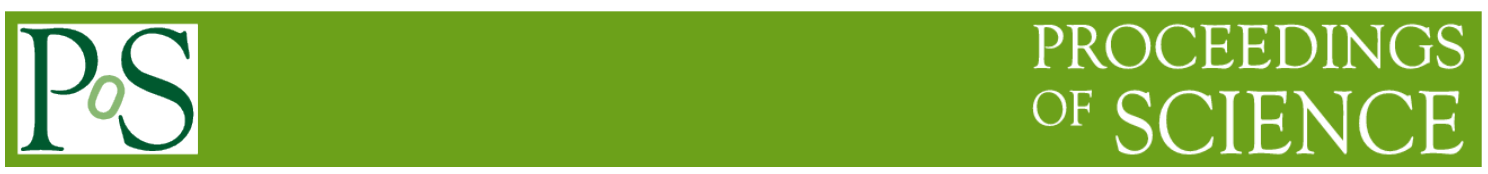

\title{
A Base Sleep Mechanism for Minimizing Energy Cost
}

\section{Dongsheng Han ${ }^{12}$}

North China Electric Power University, Baoding, 071003, China

E-mail:handongsheng@ncepu.edu.cn

\section{Bing Zheng ${ }^{3}$}

North China Electric Power University, Baoding, 071003, China

E-mail:z_bing80230163.com

\section{Zhixiong Chen}

North China Electric Power University, Baoding, 071003, China

E-mail:chenzx1983esohu.com

In order to mitigate the economic burden of Mobile Network Operators (MNOs) and restrict the emission of $\mathrm{CO}_{2}$, a joint power management model powered by hybrid sources is established. It connects wireless communication system and smart grid by setting price factor reasonably. Besides, a based sleep mechanism named as Energy Cost First (ECF) algorithm is proposed to minimize the energy cost of base station system by deploying the cheapest energy source. The simulation results show that the proposed algorithm can achieve nearly two-thirds energy cost reductions in comparison with the conventional scheme although it consumes a little more electricity. ECF algorithm makes full advantage of renewable energy to reduce the practical power consumption of traditional energy, as a result, it can effectively reduce the expenditure on energy in the wireless communication system and is consistent with the interests of MNOs.

CENet2017

22-23 July 2017

Shanghai, China

\footnotetext{
${ }^{1}$ Speaker

${ }^{2}$ This study is supported by The National Natural Science Foundation of China(No. 61601182 , No.61302106), Natural Science Foundation of Hebei Province

(No.F2017502059 , No.F2014502029), the Fundamental Research Funds for the Central Universities (No. 2016MS98)

${ }^{3}$ Corresponding Author
} 


\section{Introduction}

The mobile network throughput needs to increase by a factor of 1000 in the next 10-15 years [1]. The cellular operators have to deploy dense network to meet the challenge for consistent quality of service (QoS) and high data rate. The dense network employs ultra-dense and irregular operator-deployment of low-power and low-cost base stations (BSs) [2]. The explosive growth of mobile users and BSs has exacerbated the power consumption and aggravated the emission of $\mathrm{CO}_{2}$, which leads to a series of economic and environmental issues. The BS side consumes over $70 \%$ electricity in the wireless communication system [3]. The energy efficiency, defined as the throughput per Joule, is a popular metric to evaluate system performance [4-7]; however, the expenditure of communication bases operation is the major concern that the Mobile Network Operators focus on. Besides, the traffic load of mobile network features the space-time variation characteristic due to users' mobility and daily routine. There is a broad consensus that deploying sleep mechanism to shut down the low-traffics BSs during off-peak period to achieve the goal of energy saving [8-11]. As the renewable energy generator can harvest energy from the surroundings and the price of renewable energy is much cheaper than that of the traditional energy, it has become another approach to relieve the financial stress [12]. A cooperative energy trading model in coordinated multipoint (CoMP) system was presented, which makes full use of renewable energy [13]. Furthermore, the failure cascade in interdependent network was investigated such as the coupled communication network and the power grid network [14]. It is necessary to consider the coupling preference when designing robust interdependent network [15].

However, the above literature did not address the relationship between the energy cost and the power consumption while the mobile of user, as an important characteristic in wireless network, was neglected. Furthermore, the energy cost due to power consumption has been a focus of concern and improvement. Considering all the above, a based sleep mechanism named ECF algorithm, based on the joint power management model, is proposed from the economical aspect. It describes the relationship by setting reasonable energy price and reduces considerable energy cost.

\section{Joint Power Management Model}

\subsection{Communication System Model}

We consider a coordinated system with joint processing BSs, the number $N$ of which satisfying $N>1$. Each base is equipped with $M>1$ antennas of the transmitting power $P_{t}$ and is powered by independent renewable energy generator. The supply rate of the renewable energy varies from BS to BS because of difference of the corresponding generators. The mobile terminals (MTs) are equipped with the single antenna of received power $P_{r}$ and the number is denoted as $K$, which are randomly generated in the BSs coverage range. For the sake of convenience, we denote the set of BSs and that of MTs as $\mathbf{N}=\{1, \ldots, N\}$ and $\mathbf{K}=\{1, \ldots, K\}$, whereas $N^{\prime}$ and $\bar{N}^{\prime}$ are the number of active and the sleeping BSs.

In order to jointly reduce the energy cost and the power consumption, a sleep mechanism to turn the redundant BSs into sleep mode has been invested. Suppose that the system with two 
active BSs, denoted as $B S_{1}$ and $B S_{2}$, can meet the users' requirements. Then the received signal for MT $k$ can be expressed as

$$
y_{k}=h_{k} x_{k}+\sum_{k \neq l} h_{k} x_{l}+\sigma^{2}
$$

where $h_{k} \in C^{1 \times 2 \mathrm{M}} \quad$ refers to the Rayleigh fading channel vector for MT $k, \quad x_{k} \in C^{2 \mathrm{M} \times 1}$ refers to the pre-coding signal, and $\sigma^{2}$ refers to the background additive white Gaussian noise at MT $k$. Considering large scale fading, the channel vector for MT $k$ can be expressed as

$$
\begin{aligned}
h_{k} & =\left[\sqrt{P r_{1 \mathrm{k}}} h_{1 \mathrm{k}} \sqrt{P r_{2 \mathrm{k}}} h_{2 \mathrm{k}}\right] \\
& =\left[\sqrt{P r_{1 \mathrm{k}}} h_{1 \mathrm{k} 1} \sqrt{P r_{1 \mathrm{k}}} h_{2 \mathrm{k} 2} \ldots \sqrt{P r_{1 \mathrm{k}}} h_{1 \mathrm{kM}} \sqrt{P r_{2 \mathrm{k}}} h_{2 \mathrm{k} 1} \sqrt{P r_{2 \mathrm{k}}} h_{2 \mathrm{k} 2} \ldots \sqrt{P r_{2 \mathrm{k}}} h_{2 \mathrm{kM}}\right]
\end{aligned}
$$

where $P r_{1 k}, P r_{2 k}$ is the received power for MT $k$ from $B S_{1}$ and $B S_{2}$.

We assume that $h_{k}, \quad \forall k \in K \quad$ is available at transmitter, hence, the zero-forcing (ZF) precoding can be used to eliminate the inter-user interference, that is

$$
x_{k}=v_{k} s_{k}
$$

where $\boldsymbol{v}_{\mathrm{k}}$ is the pre-coding vector for MT $k$, and $s_{k}$ is the transmitting signal. Thus, the signal-to- interference-plus-noise ratio (SINR) at MT $k$ is given by

$$
\operatorname{SINR}_{k}=\frac{\left|h_{k}{ }^{H} v_{k}\right|^{2}}{\sigma^{2}}
$$

With $N^{\prime}>1$, we use a joint channel vector $h_{k}$ to calculate the pre-coding vector $\boldsymbol{v}_{k}$, where the channel vector $h_{k}$ is normalized firstly

$$
\begin{gathered}
g_{k}=\frac{h_{k}}{\left\|h_{k}\right\|} \\
v_{k}=g_{k}{ }^{\prime} \cdot \operatorname{inv}\left(g_{k} * g_{k}{ }^{\prime}\right)
\end{gathered}
$$

\subsection{Power Consumption Model}

Any of the BSs can be powered by the smart grid (i.e. traditional energy) and the renewable energy (including wind energy and solar energy). The generation cycle $\omega_{t}$ of new energy has been averaged over 15 minutes. There are 96 time periods over 24 hours. Besides, the price of traditional energy $q_{n, t}$ varies with time period because of the peak-valley TOU tariff strategy. Note that the electricity price of grid repurchases renewable energy is $q_{b a c k}$, which satisfies $0<q_{\text {back }}<q_{n, t}$.

Paper [8] shows that the solar energy generation rate follows a similar pattern as normal distribution which can be expressed as

$$
\text { energy }_{\text {solar }}(t)=\lambda_{s} \exp \left(\frac{-(t-48)^{2}}{81}\right), \lambda_{s} \in\left\{\begin{array}{ll}
0.7 & 0.3
\end{array}\right\}
$$

and the wind generation rate is fixed as

$$
\text { energy }_{\text {wind }}(t)=\lambda_{w}, \lambda \in\left\{\begin{array}{ll}
0.6 & 0.3
\end{array}\right\}
$$

Statistical data can be used to indicate the probability of weather conditions, then determine the different values of $\lambda_{s}, \lambda_{w}$. According to the statistics, the probabilities of sunny day and cloudy day in a year are denoted as $\left\{P_{\text {sun }} P_{\text {cloud }}\right\}=\{0.60 .4\}$, and the probabilities of gale and breeze are denoted as $\left\{P_{\text {gale }} P_{\text {brezze }}\right\}=\{0.40 .6\}$. 
In the cellular system, the power consumption for active BSs and sleeping BSs are denoted as $P_{\text {active, },}$ and $P_{\text {sleep }, t}$. The power consumption at active BS typically includes the transmitting power because of power amplifiers (PA) and the fixed power because of other components such as cooling systems and base-band units [9]. We denote the transmitting power of each antenna for the period $t$ as $\quad P_{t x, t} \geq 0$ and the fixed power as a constant denoted by $P_{f i x}$. Then we obtain the power consumption for time period $t$ at $\mathrm{BS} n$, denoted as $P_{B S, t}$, which can be expressed as [13]

$$
\begin{aligned}
& P_{B S, t}=P_{\text {active }, t}=P_{f i x}+\eta P_{t x, t}, \text { if } \mathrm{BS} \text { in active state } \\
& \text { or }=P_{\text {sleep }, t}, \quad \text { if } \mathrm{BS} \text { in sleep state }
\end{aligned}
$$

where $\quad \eta>0$ is a constant representing the PA efficiency.

\section{Problem Formulation and ECF Algorithm}

\subsection{Problem Formulation}

We aim at optimizing the resource allocation in energy domain subjected to each MT's QoS. A based sleep mechanism named ECF algorithm is presented to minimize the energy cost of base station system by deploying the cheapest energy source. The BS group corresponding to the minimum cost will turn to be active for each period time. For ECF algorithm, the traditional energy and renewable energy are the candidates to supply the system. Note that the equipping BSs with energy storage devices and non-ideal hardware should be further studied.

Mathematically, we formulate ECF algorithm as follows. $P_{n, t}$ and $P_{r, t}$ are the electricity supply of traditional energy and renewable energy, and $P_{e, t} \geqslant 0$ is the remaining electricity of renewable energy in period $t$. Then, we formulate the energy cost minimizing problem as

$$
\min \text { Price } 1=\min \sum_{n \in N} \sum_{t \in \Gamma}\left(q_{n, t} P_{n, t}-q_{\text {back }} P_{e, t}\right)
$$

And (3.1) can be decomposed into the energy cost in each period like (3.2), which can be reformulated as

$$
\begin{gathered}
\min \text { price }_{t}=\min \sum_{n \in N}\left(q_{n, t} P_{n, t}-q_{\text {back }} P_{e, t}\right) \\
\text { s.t. } \quad \sum_{n \in N}\left(P_{r, t}+P_{n, t}\right)=\sum_{n \in N^{\prime}} P_{a c t i v e, t}+\sum_{n \in \bar{N}^{\prime}} P_{\text {sleep }, t}, \quad \forall t \in \Gamma \\
P_{e, t}=P_{r, t}-P_{B S, t}, \quad \text { if } P_{r, t} \geqslant P_{B S}, \forall t \in \Gamma \\
P_{r, t} \leqslant \text { energy }_{r e} \times \omega_{t} \\
P_{t x, t} \leqslant P_{t x-\max }, \forall t \in \Gamma \\
\operatorname{SINR}_{k} \geqslant \operatorname{SINR}_{l}, \forall k \in K
\end{gathered}
$$

The constraint in (3.3) ensures that the power output is equal to the power input in period $t$. (3.4) is for calculating the remaining renewable energy. And the available value of renewable energy for period $t$ can be calculated by (3.5) taking advantage of (2.7) and (2.8). (3.6) and (3.7) denote the transmitting power constraint for period $t$ and the QoS constraint for MT $k$.

Specially, the conventional scheme is obtained when the renewable energy is ignored, for which the issue of minimizing energy cost is equivalent to that of minimizing power consumption, and can be given as

$$
\min P=\min \sum_{t \in \Gamma}\left(\sum_{n \in N^{\prime}}\left(\eta P_{t x, t}+P_{f i x}\right)+\sum_{n \in \bar{N}}, P_{\text {sleep }}\right)
$$

If $q_{\text {low }, t}=\min \left(q_{n, t}, q_{b}\right)$, the corresponding energy cost can be expressed as 


$$
\text { Price } 2=\sum_{t \in \Gamma} q_{\text {low }, t}\left(\sum_{n \in N^{\prime}}\left(\eta P_{t x, t}+P_{f i x}\right)+\sum_{\text {ninbar } N^{\prime}} P_{\text {sleep }}\right)
$$

s.t. (3.6) - (3.7)

Obviously, the diversity of energy is ignored when the traditional energy adopting peakvalley TOU tariff strategy is treated as the only source. In this paper, we take advantage of the energy at low price such as renewable energy to supply, which conforms to the dominant trend. The grid that repurchases the remaining renewable energy can further reduce the energy cost.

\section{Simulation Results}

We consider a practical five-BS wireless communication system which deploys the pattern of joint processing (with $N=5$ ), denoted as $n=1,2, \ldots, 5$. Each BS is equipped with the transmitting antennas $M=4$. Without loss of the generality, assume that BS1 and BS5 are equipped with the wind generators, BS2 and BS4 have solar generators, whereas BS3 equips both generators. Different choices of renewable energy generator mean different rates of supply. To describe the users' mobility characteristic, we randomly generate users (i.e., $K=8$ ) with single antenna at each period of time. Corresponding to the above system model, assume only two active BSs (i.e., $\mathrm{N}^{\prime}=2$ ) and $\eta=10$. The fixed consumption of active BS and sleep consumption are $P_{f i x}=500 \mathrm{~W}$ and $P_{\text {sleep }}=250 \mathrm{~W}$. The constraint of transmitting power is $P_{t, t,}=25 \mathrm{~W}$, while the noise power is $-50 \mathrm{dBm}$.

The traditional energy deploys the peak-valley TOU tariff strategy and set the electricity price factor as $\alpha$. The related documents show that 9:00-12:00 and 17:00-22:00 are the peak period, when the price is $1.33 \alpha / \mathrm{kWh} .23: 00 \mathrm{pm}-8: 00 \mathrm{am}$ of the next day is the valley period, when the price is $0.51 \alpha / k W h$. The rest are medium period of which the price is $0.87 \alpha / k W h$.

Fig. 1 shows the average consumption of each period. For the conventional scheme, the power consumption remains constant over time without significant fluctuations because the conventional scheme determines active BS group by monitoring which one has the minimum power consumption regardless of the energy cost. In contrast, ECF algorithm, the resulting average consumption during each period of time is observed to vary, following a similar pattern as the corresponding generate rate of renewable energy. For example, during $t=38-52$, the sum generate rate of solar energy and wind energy is greater and the renewable energy rate is the largest rate in a day when $t=48$, the consumption excesses the others and reaches a maximum when $t=48$. Furthermore, the total power consumption is illustrated in Fig. 2, which can be observed that ECF algorithm leads to a slight increase by $1.75 \%$.

Fig. 3 describes the average price of each time, which has a close connection with the price of traditional energy during each period of time. Besides, the generate rate of renewable energy will influence the average price of ECF algorithm. Taking $t=37-60$ for example, the curve is concave with a contrary pattern of renewable energy rate. Fig. 4 illustrates the total energy cost of the base station system. The slope of the conventional scheme is positively related to the price of traditional energy. ECF algorithm takes full advantage of renewable energy and determines active BSs by choosing the cheapest group. It can achieve $63.29 \%$ total energy cost reductions over the conventional scheme. 

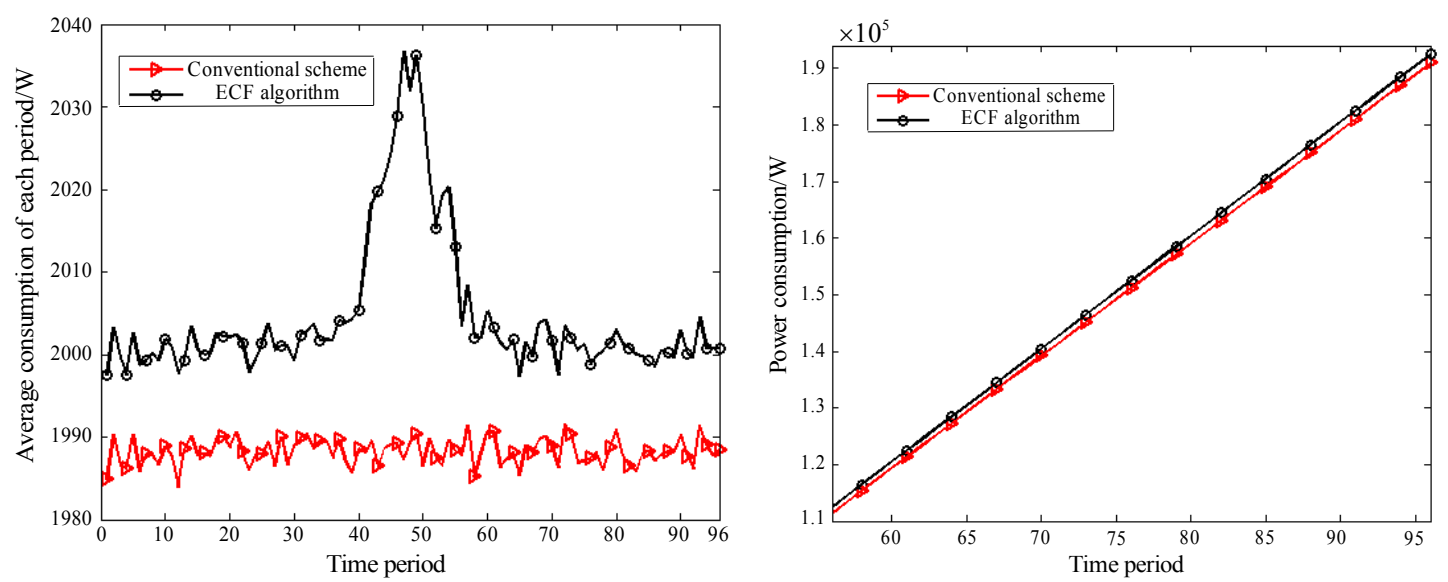

Figure 1: Average Consumption during Each Period Figure 2:Power Consumption

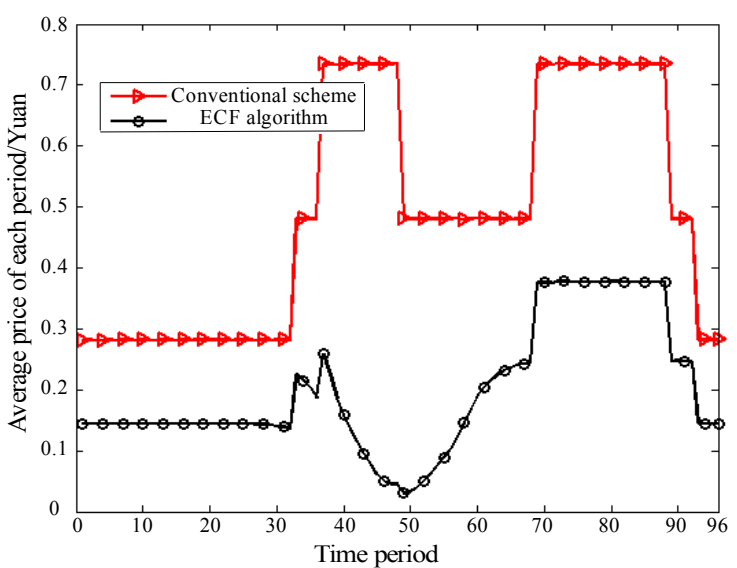

Figure 3:Average Price during Each Period

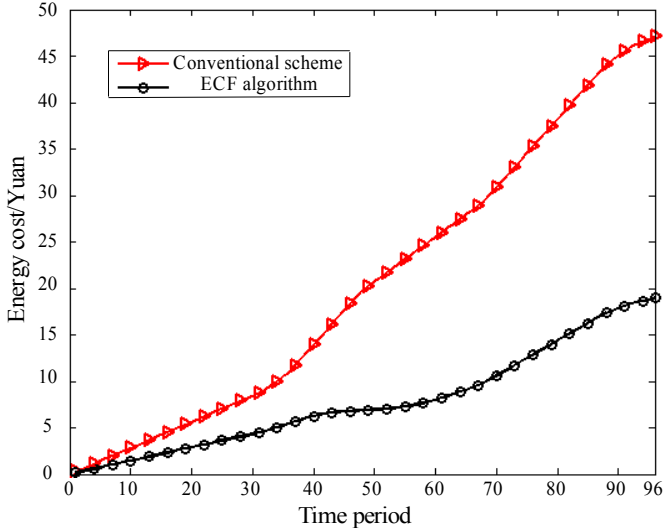

Figure 4:Energy Cost

\section{Conclusion}

In this paper, a joint power management model is established to connect wireless communication system and smart grid. Besides, we propose a based sleep mechanism named ECF algorithm to reduce the energy cost of base station system in the smart grid environment. Considering the diversity of energy, we achieve a balance between the power consumption and the energy cost subject to each MT's QoS. The hybrid source which includes the traditional energy and the renewable energy can be considered as the source of each BS. ECF algorithm which takes full use of the renewable energy reaches a significant superiority and achieves considerable energy cost reductions over the conventional scheme although it leads to an acceptably fluctuation of power consumption.

\section{References}

[1] I. K. Son, S. Mao, Li Y, et al. Frame-Based Medium Access Control for $5 G$ Wireless Networks[J]. Mobile Networks and Applications. 20(6):763-775(2015)

[2] E. Björnson, L. Sanguinetti, M. Kountouris. Deploying Dense Networks for Maximal Energy Efficiency: Small Cells Meet Massive MIMO[J]. IEEE Journal on Selected Areas in Communications. 34(4):832-847(2015) 
[3] C. Han, T Harrold, S. Armour, et al. Green radio: radio techniques to enable energy-efficient wireless networks[J]. IEEE Communications Magazine. 49(6):46-54(2011)

[4] Y. Hu, B. Ji, Y. Huang, et al. Energy-efficiency resource allocation of very large multi-user MIMO systems[J]. Wireless Networks. 20(6):1421-1430(2014)

[5] K. Son, H. Kim, Y. Yi, et al. Base Station Operation and User Association Mechanisms for Energy-Delay Tradeoffs in Green Cellular Networks[J]. IEEE Journal on Selected Areas in Communications. 29(8):1525-1536(2011)

[6] S. K. Mohammed. Impact of Transceiver Power Consumption on the Energy Efficiency of ZeroForcing Detector in Massive MIMO Systems[J]. IEEE Transactions on Communications. 62(11):3874-3890(2014)

[7] A. Bousia, E. Kartsakli, A. Antonopoulos, et al. Multiobjective Auction-Based Switching-Off Scheme in Heterogeneous Networks: To Bid or Not to Bid? [J]. IEEE Transactions on Vehicular Technology. 65(11):9168-9180(2016)

[8] S. Han, C. Yang, A. F. Molisch. Spectrum and Energy Efficient Cooperative Base Station Doze[J]. IEEE Journal on Selected Areas in Communications. 32(2):285-296(2014)

[9] E. Oh, B. Krishnamachari. Energy Savings through Dynamic Base Station Switching in Cellular Wireless Access Networks[C], in Proc. Global Telecommunications Conference. IEEE.1-5(2010)

[10]S. Q. Han, C. Y. Yang, G. Wang, and M. Lei, On the energy efficiency of base station sleeping with multicell cooperative transmission[C], in Proc. IEEE PIMRC.1536-1540(2011)

[11] K Son, H Kim, Y Yi, et al. Base Station Operation and User Association Mechanisms for Energy-Delay Tradeoffs in Green Cellular Networks[J]. IEEE Journal on Selected Areas in Communications. 29(8):1525-1536(2011)

[12] T Han, N Ansari. Power Mobile Networks with Green Energy. IEEE Wireless Commun. 21,9096(2014)

[13]J Xu, R Zhang. Cooperative energy trading in CoMP systems powered by smart grids[C]. IEEE Global Communications Conference. IEEE. 2697-2702(2014)

[14]S Hong, C Lv, T Zhao, et al. Cascading failure analysis and restoration strategy in an interdependent network[J]. Journal of Physics A Mathematical \& Theoretical. 49(19): 195101(2016)

[15]S Hong, B Wang, X Ma, et al. Failure cascade in interdependent network with traffic loads[J]. Journal of Physics A Mathematical \& Theoretical. 48(48):485101(2015) 